doi: 10.2306/scienceasia1513-1874.2013.39S.031

\title{
Modelling the spatiotemporal dynamics of diffusive prey-predator interactions: Pattern formation and ecological implications
}

\author{
Mohd Hafiz Mohd ${ }^{\mathrm{a}, \mathrm{b}, *}$, Yahya Abu Hasan ${ }^{\mathrm{a}}$ \\ ${ }^{a}$ School of Mathematical Sciences, Universiti Sains Malaysia, 11800 USM, Penang, Malaysia \\ b Department of Mathematics and Statistics, University of Canterbury, Private Bag 4800, Christchurch 8140, \\ New Zealand \\ *Corresponding author, e-mail: mohdhafizmohd@gmail.com, hafiz.mohd@pg.canterbury.ac.nz
}

Received 7 Jan 2013

Accepted 5 Apr 2013

\begin{abstract}
In this paper, we investigate the formation of spatiotemporal patterns in ecological systems via numerical simulations. We study the diffusive prey-predator model and observe the rich dynamical structure as a result of preypredator interactions in one and two spatial dimensions. In 1-d numerical investigations we show that, for a large class of initial conditions, the evolution of the model leads to the emergence of non-stationary irregular patterns, given that the local kinetics of the system is oscillatory. This corresponds to spatiotemporal chaos. The irregular patterns first emerge inside a subdomain of the system. This subdomain then steadily grows with time and, finally, the irregular chaotic patterns invade the whole space, displacing the regular pattern. For 2-d numerical simulations we observed the formation of spiral waves, patchy structures, spiral defect chaos and spatiotemporal chaos due to Turing instability in our prey-predator model. These two-dimensional patterns are very beautiful and very interesting to be observed and interpreted from the ecological point of view.
\end{abstract}

KEYWORDS: reaction-diffusion equations, local stability analysis, spatiotemporal chaos, spiral defect chaos, diffusiondriven instability

\section{INTRODUCTION}

The study of complex population dynamics involving a prey-predator interaction has received much attention since the early days of ecology. Lotka and Volterra independently developed a simple model of interacting species ${ }^{1,2}$. There are several types of interaction between species, namely, competition, mutualism and prey-predator. But among these three interactions, the dynamic relationship between predators and their prey has long been and will continue to be one dominant research area in mathematical ecology owing to its importance and universal existence ${ }^{3}$.

Generally, the prey-predator model obeys two fundamental principles: one is that population dynamics can be decomposed into birth and death processes; and the other one is the conservation of mass principle, stating that predators can only grow only as a function of what they have eaten ${ }^{4}$. Hence the basic prey-predator interaction can be described by using a system of ordinary differential equations which model the spatial distribution of species as a function of time.

However, the presence of a diffusion mechanism in prey-predator interaction, which is considered as the principal process of motion, changes the behaviour and nature of the whole model. It is now a partial differential equation, which can be categorized as a reaction-diffusion system. The diffusive prey-predator model has been studied extensively in Refs. 5-7.

The inclusion of diffusion terms has made our prey-predator model more complicated and it is very difficult to analyse and solve analytically. According to Ref. 8 the non-uniform stationary state of a preypredator model, which corresponds to the spatiotemporal patterns, cannot be found analytically. Thus many scientists resort to numerical simulations to study the behaviour of the system. If the model parameters are selected properly, the numerical simulations will give rise to rich spatiotemporal dynamical structure in one-dimensional space. If we consider 2-d numerical simulations, it will result in two-dimensional patterns, namely, homogeneous distributions, stationary patterns (striped, spotted, or both), emergence of spatiotemporal chaos and spiral waves.

Many researchers have performed analytical and numerical investigations of diffusive prey-predator 
model from different aspects: e.g., the spatiotemporal dynamics of multi-species host-parasitoid interactions and its ecological effects ${ }^{9}$, the role of diffusion coefficients on Turing instability in a prey-predator system ${ }^{10}$. Other scientists have also studied the preypredator problems extensively: for instance, the problem of biological invasion in prey-predator model and the dynamics of population through non-Turing pattern formation with specified initial conditions ${ }^{11-13}$.

In this paper, we study the rich dynamical structure as the result of prey-predator interactions in one and two-space dimensions. In particular, we investigate the spatiotemporal pattern of diffusive prey-predator model and the emergence of irregular chaotic patterns. We also investigate the occurrence of diffusion-driven instability (Turing instability or diffusive instability). A necessary condition to observe the Turing instability is that the predator must diffuse faster than the prey.

\section{MATHEMATICAL MODEL}

We consider

$$
\begin{aligned}
& u_{t}=\alpha \Delta u+F(u, v) \\
& v_{t}=\beta \Delta v+G(u, v)
\end{aligned}
$$

where $u$ and $v$ represent the prey and predator population densities, $\alpha$ and $\beta$ are diffusion coefficients of prey and predator, respectively, and $\Delta$ is the Laplacian. We analyse the above model under the initial conditions

$$
\begin{gathered}
u(x, y, 0)=f(x, y) \\
v(x, y, 0)=g(x, y),
\end{gathered}
$$

with $x, y \in \Omega$. Equations (1) are subject to reflective boundary conditions (i.e., Neumann boundary conditions):

$$
\frac{\partial u}{\partial n}=\frac{\partial v}{\partial n}=0, \quad(x, y) \in \partial \Omega, t>0
$$

where $\mathbf{n}$ is the outward normal to $\partial \Omega$.

We assume that the local growth of the prey species is logistic and the predator community displays the Holling type II functional response. Hence

$$
\begin{aligned}
F(u, v) & =\frac{\varphi}{\gamma} u(\gamma-u)-\eta \frac{u v}{u+H} \\
G(u, v) & =\kappa \eta \frac{u v}{u+H}-m v
\end{aligned}
$$

where $\varphi$ is the maximum growth rate of the prey population, $\gamma$ is the carrying capacity for the prey population, and $H$ is the half-saturation density for the prey population.
Let

$$
\bar{u}=\frac{u}{\gamma}, \bar{v}=\frac{v \eta}{\varphi \gamma}, \bar{t}=\varphi t, \delta=\frac{H}{\gamma}, \mu=\frac{m}{\varphi}, \varepsilon=\frac{\kappa \eta}{\varphi} .
$$

Then

$$
\begin{aligned}
& F(\bar{u}, \bar{v})=\bar{u}(1-\bar{u})-\frac{\bar{u} \bar{v}}{\bar{u}+\delta} \\
& G(\bar{u}, \bar{v})=\frac{\varepsilon \bar{u} \bar{v}}{\bar{u}+\delta}-\mu \bar{v} .
\end{aligned}
$$

After dropping the bars, our diffusive prey-predator model becomes

$$
\begin{aligned}
& u_{t}=\alpha \Delta u+u(1-u)-\frac{u v}{u+\delta} \\
& v_{t}=\beta \Delta v+\frac{\varepsilon u v}{u+\delta}-\mu v .
\end{aligned}
$$

There are three positive equilibrium states. Two of them are trivial: $(0,0)$ corresponds to total extinction of prey-predator population; $(1,0)$ indicates the persistence of prey and extinction of predator population. Both are saddle points. The non-trivial equilibrium state is $(\hat{u}, \hat{v})$ where

$$
\begin{aligned}
& \hat{u}=\frac{\delta \mu}{\varepsilon-\mu}, \quad \varepsilon>\mu \\
& \hat{v}=(1-\hat{u})(\hat{u}+\delta), \quad \delta<\frac{\varepsilon-\mu}{\mu} .
\end{aligned}
$$

We start by choosing $\delta=0.4, \varepsilon=2$, and $\mu=0.8$ in which case $(\hat{u}, \hat{v})=\left(\frac{4}{15}, \frac{22}{45}\right)$ and standard linear stability analysis shows that this is an unstable spiral.

\section{NUMERICAL SIMULATIONS}

In this section, we look at 1-d simulations. For simplicity of computation, we choose $\alpha=1$. We use $\beta=1$ so as to exclude the possibility of Turing instability. We use the initial conditions

$$
\begin{aligned}
& u(x, 0)=\hat{u} \\
& v(x, 0)=\hat{v}+\phi x-\sigma .
\end{aligned}
$$

We can see that the two populations oscillate which indicates the unstable behaviour for the prey and predator populations (Fig. 1). There is no indication of irregular (chaotic) dynamics.

When $\phi$ is small, the initial conditions evolve to a smooth non-monotonic spatial distributions of species, and this results in the prey-predator densities as in Fig. 1. Now, let us try to change $\phi$ and $\sigma$ as illustrated in Fig. 2.

The initial conditions (8) with $\phi=2 \times 10^{-5}$ and $\sigma=4 \times 10^{-2}$ leads the system to evolve into an irregular non-stationary pattern. And if we plot the 


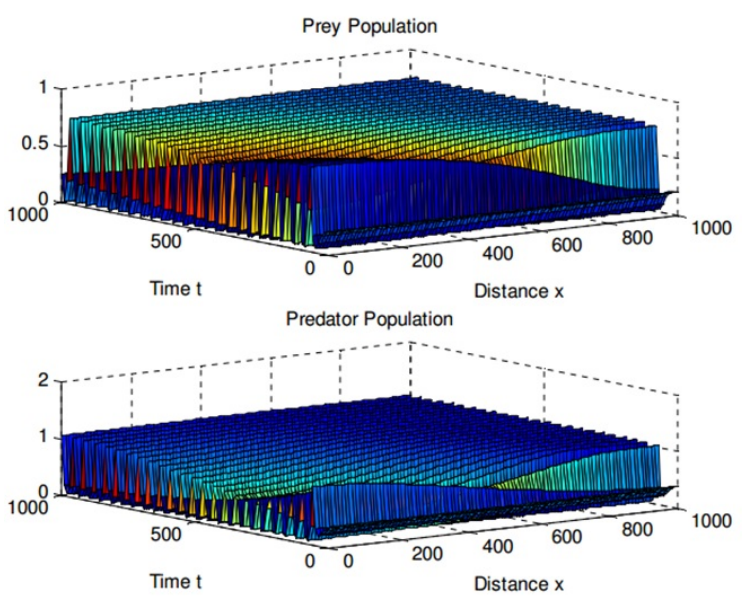

Fig. 1 The one-dimensional approximate prey-predator densities using $\phi=10^{-5}$ and $\sigma=10^{-2}$. Parameter values are $\delta=0.4, \varepsilon=2$ and $\mu=0.8$, with zero flux boundary conditions and initial conditions as in (8).

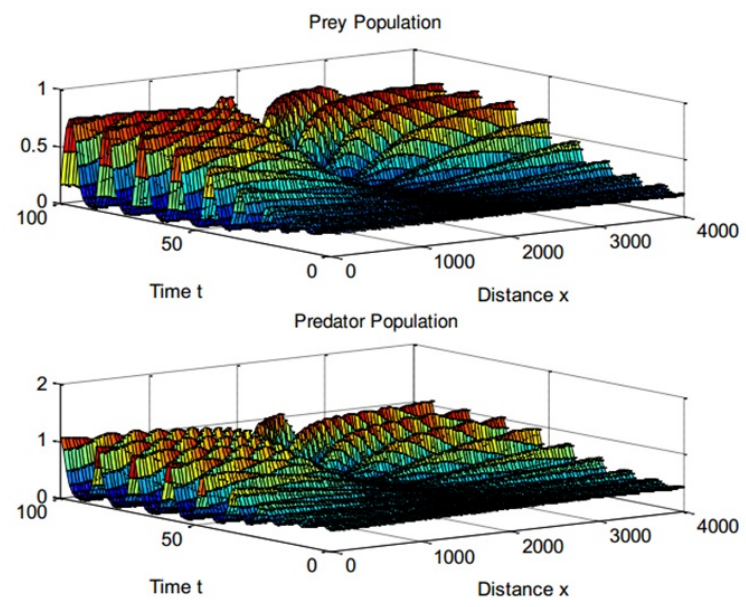

Fig. 2 The one-dimensional approximate prey-predator densities using $\phi=2 \times 10^{-5}$ and $\sigma=4 \times 10^{-2}$. Parameter values are $\delta=0.4, \varepsilon=2$ and $\mu=0.8$, with zero flux boundary conditions and initial conditions as in (8).

local phase plane of the system after the formation of the above irregular dynamics, we can observe closed trajectories as in Fig. 3. It shows the local phase plane of model (6) after the formation of an irregular non-stationary pattern as in Fig. 2. Observe that the boundary of the domain coincides with the limit cycle, or in other words the irregular spatiotemporal oscillations have invaded the local phase plane and have caused chaotic dynamics (see Ref. 13).

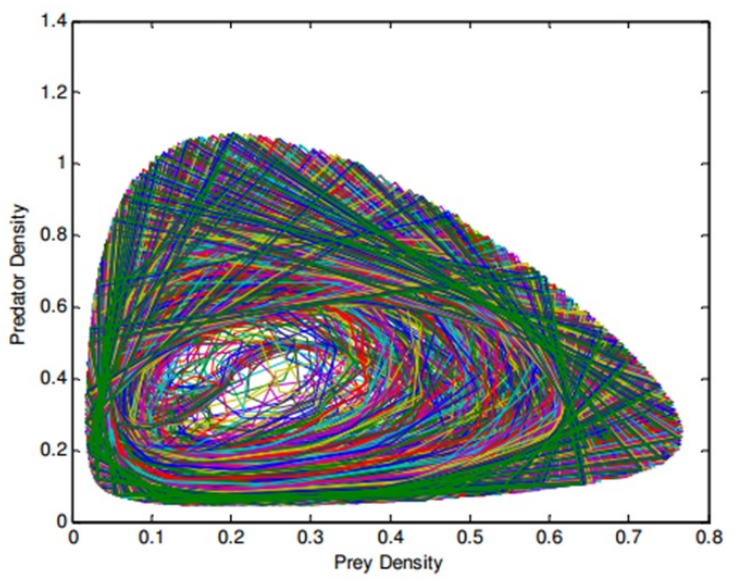

Fig. 3 Local phase plane of model (6) with initial conditions as in (8).

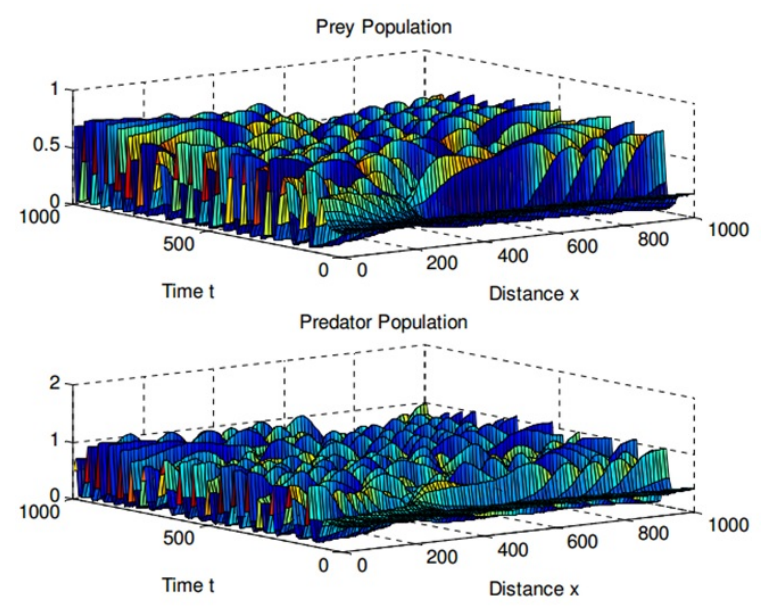

Fig. 4 The one-dimensional approximate prey-predator densities using $\beta=150$. Parameter values are $\delta=0.4$, $\varepsilon=2$ and $\mu=0.8$, with zero-flux boundary conditions and initial conditions as in (9).

We now use the initial conditions

$$
\begin{aligned}
& u(x, 0)=\hat{u} \\
& v(x, 0)=\hat{v}+0.02 \sin \left(\frac{2 \Pi(x-1500)}{100}\right) .
\end{aligned}
$$

In order to observe the formation of a diffusive instability in the 1-d space prey-predator model, let us choose $\beta=150$. Consider the 1-d approximate preypredator densities in Fig. 4 that we obtained from our numerical simulations. The analysis confirmed the existence of Turing instability. In Turing mechanism, a homogeneous steady state which is stable with respect to spatially uniform perturbations becomes unstable under the influence of diffusion, relating to 

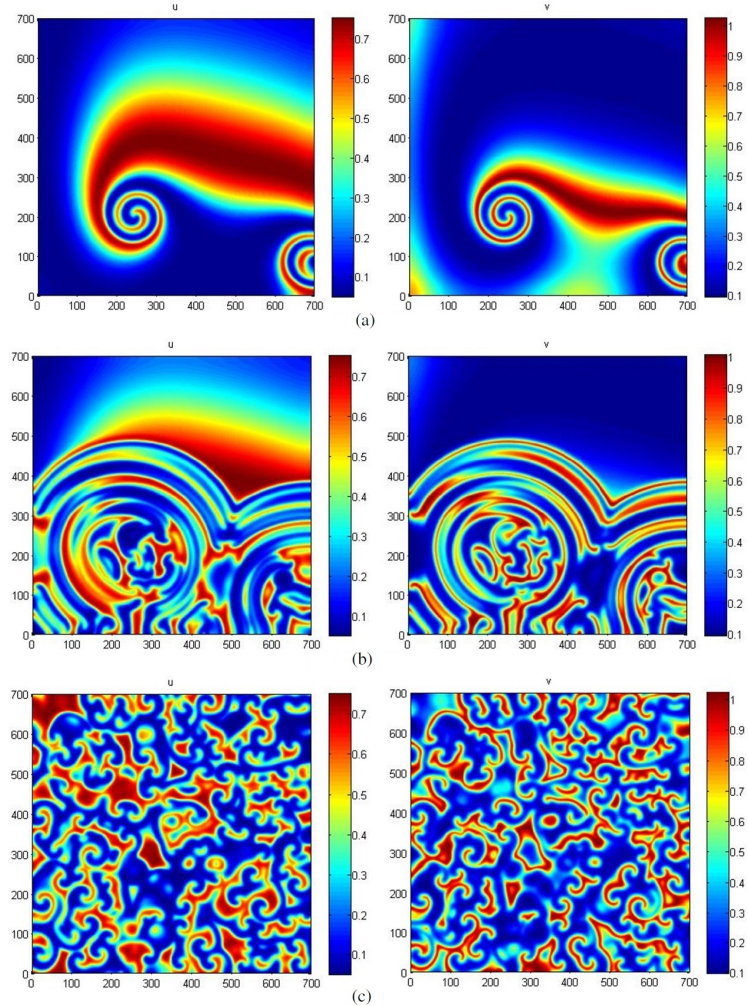

Fig. 5 Spiral pattern at times (a) $t=300$, (b) $t=1000$, (c) $t=3000$.

perturbation with appropriate spatial frequencies ${ }^{10}$. Hence these patterns show the diffusive instability from a numerical point of view.

For the 2-d model, the initial conditions are

$$
\begin{aligned}
u(x, y, 0)=\hat{u} & -\left(2 \times 10^{-7}\right)(x-0.1 y-225) \\
& \times(x-0.1 y-675) \\
v(x, y, 0)=\hat{v} & -\left(3 \times 10^{-5}\right)(x-450) \\
& -\left(1.2 \times 10^{-4}\right)(y-150)
\end{aligned}
$$

and are chosen so as to induce non-trivial spatiotemporal dynamics. These 'perturbed' initial conditions will also be used in order to test the sensitivity to initial conditions. As in the previous section, we will choose $\delta=0.4, \varepsilon=2$ and $\mu=0.8$.

We solve the diffusive prey-predator model (6) in two dimensions using a finite difference method, with a square numerical domain $L \times L$ where $L=700$. We fix the initial conditions as in (10) and $\beta=1$.

Consider the two-dimensional approximate preypredator densities in Fig. 5 that we obtained from our numerical simulations by increasing the time of experiments. In Fig. 5a we observe the formation of spirals which confirms the local stability analysis result that we obtained. After the spirals form, they grow slightly for a certain time and later their spatial structure becomes more distinct. Then, these spirals separate into two circles. Notice that the destruction of the spirals begins in their centres (Fig. 5b). These circles steadily grow bigger and when $t=3000$, we observe the emergence of patchy structure inside these circles (Fig. 5c). The blue and green coloured regions indicate the areas of low population density while the red and orange coloured regions represent the areas of high population density. We can see the emergence of irregular patches covering the whole domain as $t=3000$ (Fig. 5c). The patches first appear inside a subdomain of the system. This subdomain then steadily grows with time and the patchy structures invade the whole space, displacing the regular pattern.

This observation is consistent with the results reported in Refs. 7, 14. They studied the spatiotemporal dynamics in an aquatic community and discovered that the size of these patches is related to the characteristic length of observed plankton patterns in the ocean. Apart from that, Ref. 9 also reported similar results on the formation of patchiness phenomenon in multispecies host-parasitoid (prey-predator) interactions. They described the appearance of patchy structures and chaos developing behind periodic waves. It is suggested that a small instability in the periodic waves causes a small asymmetry to develop and this gives rise to chaotic dynamics.

We now investigate the effect of varying parameter $\delta$ on the spatiotemporal dynamics of model (6). We will use the same parameters as in Fig. 5, and we solve model (6) in two dimensions with a square numerical domain with $L=500$. Consider the two-dimensional approximate prey-predator densities in Fig. 6 that we obtained from our numerical simulations by varying $\delta$. We choose $\delta$ in the range $0<\delta<0.5$ and see the formation of spirals (Fig. 6). The diameter of spiral pattern increases as we decrease $\delta$.

Spiral structures emerge in Fig. $6 \mathrm{c}$ but with small defects in their patterns. The zone in which defects occur appear to oscillate and these oscillations seem to continue in the stationary structures. Ref. 14 also conducted the same experiment as in Fig. 6. They studied the dynamics of system (6) in the one-dimensional case and discovered the occurrence of chaos covering almost the entire domain when $\delta=0.05$. Based on Ref. 15, spiral structures which form around some defects are known as spiral defect chaos.

Next, let us investigate the spatiotemporal dynamics of model (6) through a Turing instability. Consider the snapshots of spatiotemporal patterns in Fig. 7. As $\beta$ increases, we observe the formation of spiral 

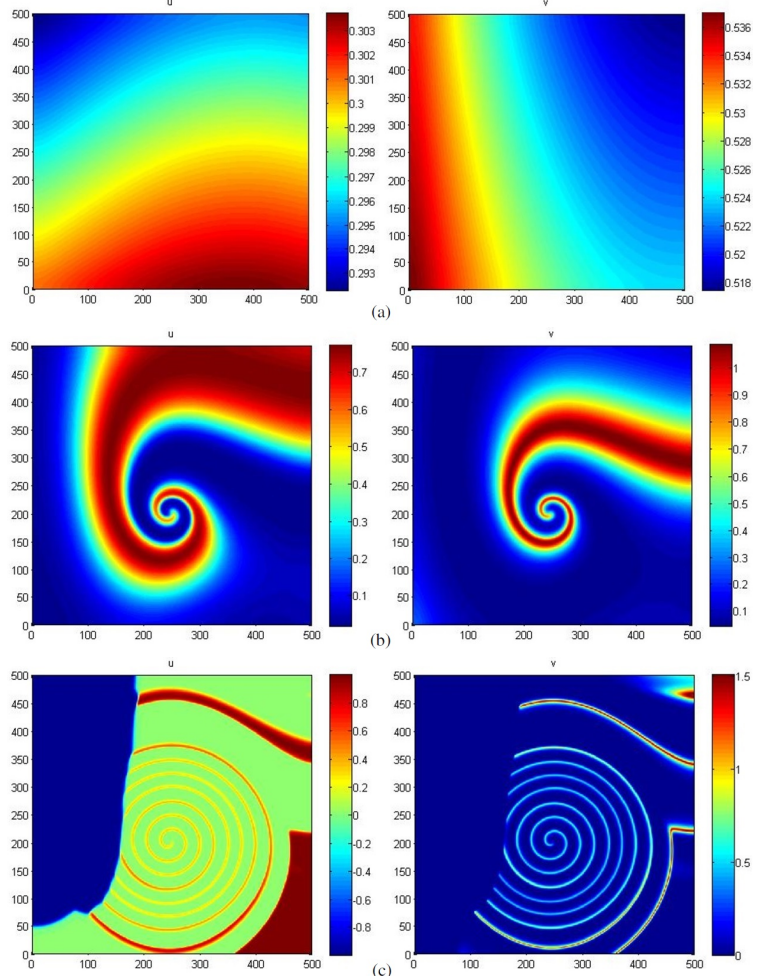

Fig. 6 Two-dimensional numerical solutions of preypredator system using finite difference discretization (timestep $\Delta t=\frac{1}{384}$ ) at $t=150$ : (a) $\delta=0.45\left(\hat{u}=\frac{3}{10}, \hat{v}=\frac{21}{40}\right)$, (b) $\delta=0.3\left(\hat{u}=\frac{1}{5}, \hat{v}=\frac{2}{5}\right)$ and (c) $\delta=0.05\left(\hat{u}=\frac{1}{30}, \hat{v}=\right.$ $\left.\frac{29}{360}\right)$.

patterns. The spiral structures get larger as we vary $\beta$ in the range of $1 \leqslant \beta \leqslant 15$.

When we change $\beta$ to 50 , we can see in Fig. 8 a that the spiral pattern loses its stability. In order to verify this situation, we increase $\beta$ to 100 (Fig. 8b). Consequently, we observe that the dynamics becomes unstable and spatiotemporal chaos develops.

Comparing the snapshots, we can see that the formation of chaotic spiral patterns is caused by the Turing instability. Turing ${ }^{16}$ considered reactiondiffusion equations of two chemicals (morphogens) and found that diffusion could destabilize an otherwise stable equilibrium. This leads to non-uniform spatial patterns. Since we fix $\alpha=1$, Turing instability will occur with large $\beta$.

If $\beta / \alpha$ is large enough, the prey growth rate will reach negative values and prey population will be driven by predators to a very low level in those regions. In other words, where the prey density is at the maximum, diffusion will lower the prey density at that point. Conversely, where the prey density is at the
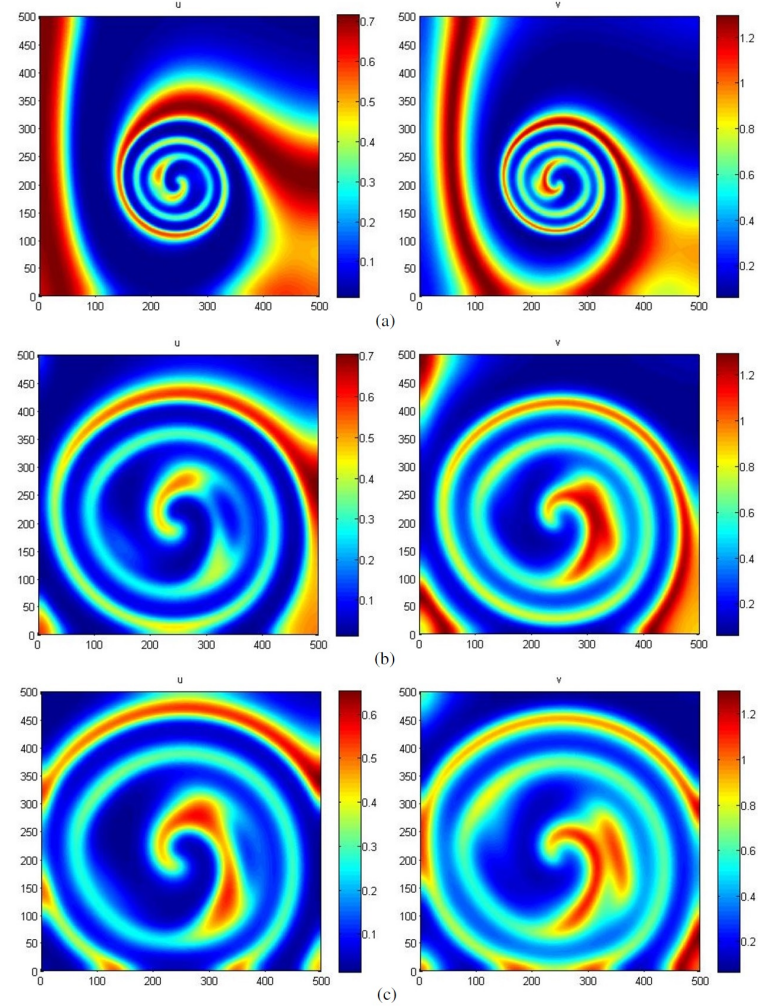

Fig. 7 Numerical solutions of 2-d prey-predator system at $t=500,\left(\hat{u}=\frac{6}{35}, \hat{v}=\frac{116}{245}\right.$ ): (a) $\beta=1$, (b) $\beta=10$ and (c) $\beta=15$.
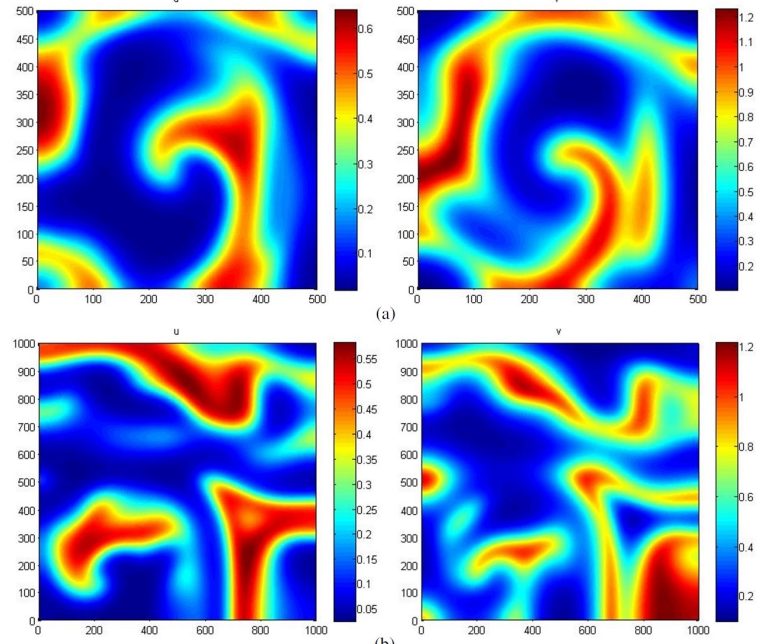

Fig. 8 Numerical solutions of 2-d prey-predator system at $t=500$ : (a) $\beta=50$ and (b) $\beta=100$.

minimum, diffusion will increase the prey density at that point ${ }^{17}$. 


\section{CONCLUSIONS}

A number of open problems remain. First, it is very interesting to investigate the formation of spatiotemporal dynamics with other choices of parameters. Next, different forms of 'disturbed' initial conditions can be considered so as to induce the non-trivial spatiotemporal patterns. Another aspect to be studied is the choice of boundary conditions; we could instead use Dirichlet and Robin boundary conditions. We expect the appearance of even more complicated phenomena using other boundary conditions. All this will help with understanding the ecological interactions between prey and predator.

Acknowledgements: This study was supported by the USM Fundamental Research Grant Scheme.

\section{REFERENCES}

1. May RM, et al (1976) Simple mathematical models with very complicated dynamics. Nature 261, 459-67.

2. Kendall BE (2001) Nonlinear dynamics and chaos. Encyclopedia of Life Sciences 13, 1-8.

3. Berryman AA (1992) The origins and evolution of predator-prey theory. Ecology 73, 1530-5.

4. Jost C (1998) Comparing predator-prey models qualitatively and quantitatively with ecological time-series data. $\mathrm{PhD}$ thesis, Institut National Agronomique ParisGrignon.

5. Murray JD (2002) Mathematical Biology, Springer.

6. Malchow H (1993) Spatio-temporal pattern formation in nonlinear non-equilibrium plankton dynamics. Proc Roy Soc Lond B 251, 103-9.

7. Medvinsky AB, Petrovskii SV, Tikhonova IA, Malchow H, Li BL (2002) Spatiotemporal complexity of plankton and fish dynamics. SIAM Rev 44, 311-70.

8. Wang W, Liu QX, Jin Z (2007) Spatiotemporal complexity of a ratio-dependent predator-prey system. Phys Rev E 75, 051913.

9. Pearce IG, Chaplain MAJ, Schofield PG, Anderson ARA, Hubbard SF (2006) Modelling the spatiotemporal dynamics of multi-species host-parasitoid interactions: heterogeneous patterns and ecological implications. J Theor Biol 241, 876-86.

10. Mukhopadhyay B, Bhattacharyya R (2006) Modeling the role of diffusion coefficients on Turing instability in a reaction-diffusion prey-predator system. Bull Math Biol 68, 293-313.

11. Sherratt JA (2001) Periodic travelling waves in cyclic predator-prey systems. Ecol Lett 4, 30-7.

12. Shigesada N, Kawasaki K (1997) Biological Invasions: Theory and Practice, Oxford Univ Press.

13. Petrovskii SV, Malchow H (2001) Wave of chaos: new mechanism of pattern formation in spatio-temporal population dynamics. Theor Popul Biol 59, 157-74.

14. Garvie MR (2007) Finite-difference schemes for reaction-diffusion equations modeling predator-prey interactions in MATLAB. Bull Math Biol 69, 931-56.

15. Bhattacharyay A (2001) Spirals and targets in reactiondiffusion systems. Phys Rev E 64, 016113.

16. Turing AM (1990) The chemical basis of morphogenesis. Bull Math Biol 52, 153-97.

17. Alonso D, Bartumeus F, Catalan J (2002) Mutual interference between predators can give rise to Turing spatial patterns. Ecology 83, 28-34. 